\title{
Technology of Ultrathin NbN and NbTiN Films for Superconducting Photodetectors
}

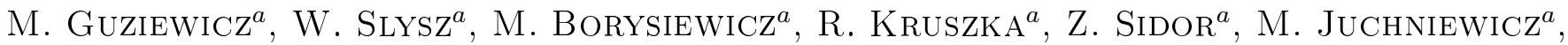 \\ K. Golaszewska ${ }^{a}$, J.Z. Domagala ${ }^{a, b}$, W. Rzodkiewicz ${ }^{a}$, J. RAtajczak ${ }^{a}$, J. BAR $^{a}$, \\ M. WEGRZECKI ${ }^{a}$ AND R. SOBOLEWSKI ${ }^{a, c}$ \\ ${ }^{a}$ Institute of Electron Technology, al. Lotników 32/46, 02-668 Warsaw, Poland \\ ${ }^{b}$ Institute of Physics, Polish Academy of Sciences, al. Lotników 32/46, 02-668 Warsaw, Poland \\ ${ }^{c}$ Departments of Electrical and Computer Engineering and Physics and Astronomy, Materials Science Program \\ and Laboratory for Laser Energetics, University of Rochester, Rochester, NY-14627-0231, USA
}

\begin{abstract}
We report fabrication and characterization of ultrathin NbN and NbTiN films designed for superconducting photodetectors. Our NbN and NbTiN films were deposited on $\mathrm{Al}_{2} \mathrm{O}_{3}$ and Si single-crystal wafers by a high-temperature, reactive magnetron sputtering method and, subsequently, annealed at $100{ }^{\circ} \mathrm{C}$. The best, $18 \mathrm{~nm}$ thick $\mathrm{NbN}$ films deposited on sapphire exhibited the critical temperature of $15.0 \mathrm{~K}$ and the critical current density as high as $\approx 8 \times 10^{6} \mathrm{~A} / \mathrm{cm}^{2}$ at $4.8 \mathrm{~K}$.
\end{abstract}

PACS: 74.62.Bf, 74.78.-w, 81.15.Cd, 81.15.Jj

\section{Introduction}

Superconducting single-photon detectors (SSPDs) based on NbN nanostripes [1, 2] have already been demonstrated to be the fastest and most sensitive optical and near-infrared photon counters $[3,4]$ and are expected to play a leading role in such applications as fiber-based quantum communications [5], optical information processing [6], free-space satellite communications [6], or medical diagnostics [7]. In a typical design, an SSPD is a $10 \times 10 \mu \mathrm{m}^{2}$ meander consisting of an ultrathin $(\approx 5 \mathrm{~nm}$ thick) and $100 \div 200 \mathrm{~nm}$ wide NbN nanostripe. The detector operates far below the $\mathrm{NbN}$ critical temperature $T_{\mathrm{C}}$ (typically $4.2 \mathrm{~K}$ ) and biased with a subcritical current [2]. Absorption of an optical photon generates a local destruction of superconductivity within the stripe (so-called hotspot formation) that grows until a resistive region is formed across the nanostripe, thus, producing a detectable voltage signal [2]. Apart from the highest possible $T_{\mathrm{C}}$, the nanostripe critical current density $J_{\mathrm{C}}$ is the second most important parameter for the best SSPD performance, as it defines the operating bias current $I_{\mathrm{b}}$. In addition, the SSPD detection efficiency has been demonstrated to be exponentially increasing function of $I_{\mathrm{b}}$ [4].

In our previous work [8], we presented our investigations on structural and superconducting transport properties of ultrathin $\mathrm{NbN}$ films deposited on various substrates suitable for both electronic and optoelectronic applications. This report is focused on the latest results of the state-of-the-art superconducting NbN films and the new fabrication thrust aimed at ultrathin NbTiN films, most recently successfully implemented in SSPDs [9].

\section{Experimental procedures}

Our NbN and NbTiN films were grown by high-temperature, reactive RF magnetron sputtering using a $\gamma-1000 \mathrm{C}$ system from the Surrey Nanosystems Ltd. The NbN films were deposited from a 3-inch diameter $\mathrm{Nb}$ target at $\mathrm{DC}$ power of $220 \mathrm{~W}$ in $\mathrm{N}_{2}-\mathrm{Ar}$ gas mixture at temperature of $850^{\circ} \mathrm{C}$. The NbTiN films were deposited from metallic $\mathrm{Nb}$ and $\mathrm{Ti}$ targets by co-sputtering. We used $\mathrm{Al}_{2} \mathrm{O}_{3}$ (0001) and $\mathrm{Si}(001)$ substrates. High purity of our films was achieved by using the $4 \mathrm{~N} 5$ purity of metallic targets, $6 \mathrm{~N}$ purity of process gases, as well as maintaining the background pressure of $10^{-8}$ mbar. The thicknesses of the studied layers varied from $6 \mathrm{~nm}$ to $20 \mathrm{~nm}$ and they were, subsequently, annealed at $\approx 1000^{\circ} \mathrm{C}$, using a rapid thermal annealing (RTA) process, in order to improve their superconducting properties.

Extensive structural characterization of our films was performed using X-ray diffraction and transmission electron microscopy (TEM) and atomic force microscopy (AFM). For high resolution XRD analysis, the PANAlytical X'Pert MRD diffractometer, equipped with the X-ray mirror, Ge (400) hybrid monochromator, and PIXCEL detector in reflected beam line, was applied. The TEM specimens were prepared by a focused ion beam (FIB) tool from Helios Nanolab and observed using the JEOL 2100 microscope. The Veeco Innova SPM AFM in- 
strument was applied to study surface roughness of our specimens.

\section{Results and discussion}

\subsection{Transport properties of $\mathrm{NbN}$ films}

Transport measurements showed that even a $6 \mathrm{~nm}$ thick NbN film was superconducting with $T_{\mathrm{C}}$ above $10 \mathrm{~K}$. The highest $T_{\mathrm{C}}=15.0 \mathrm{~K}$ was observed for $18 \mathrm{~nm} \mathrm{NbN}$ films after they were subjected to an RTA process for $20 \mathrm{~min}$.

A special nanostripe test structure was fabricated for investigation of the $J_{\mathrm{C}}$ dependence on temperature $T$, as is schematically shown in Fig. 1a. Stripes with widths varying from $600 \mathrm{~nm}$ to $15 \mu \mathrm{m}$, the fixed length of $30 \mu \mathrm{m}$, and $\mathrm{Au}$ contact pads were chosen. Figure $1 \mathrm{~b}$ shows an SEM micrograph of a $600 \mathrm{~nm}$ wide stripe. The stripe pattern was transferred from a mask by photolithography using a ma-N 1407 photoresist and reactive ion etching in the $\mathrm{CF} / \mathrm{O}_{2}$ plasma at $\mathrm{RF}$ power of $70 \mathrm{~W}$.

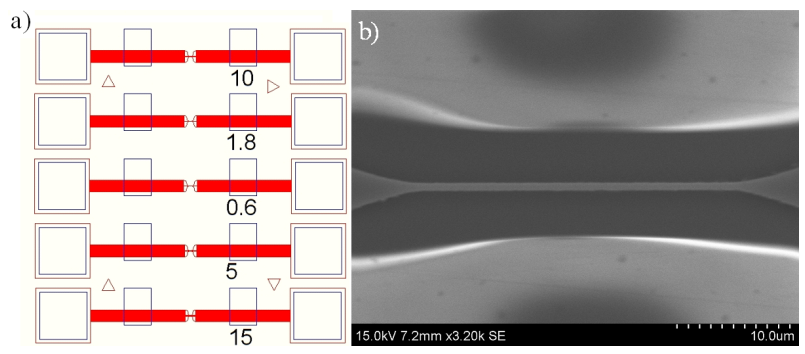

Fig. 1. (a) A photomask for a test structure used in $J_{\mathrm{C}}(T)$ studies. The numbers indicate the given stripe width in $\mu \mathrm{m}$. (b) A SEM micrograph of the narrowest (600 $\mathrm{nm}$ wide) stripe.
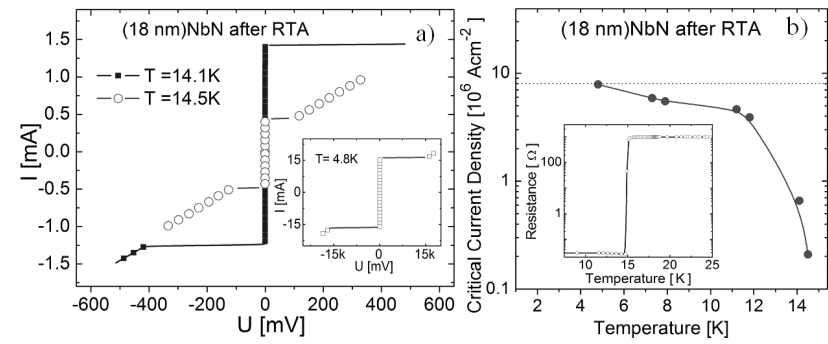

Fig. 2. (a) $I-V$ characteristics of a tested $\mathrm{NbN}$ stripe. The inset shows the $I-V$ curve taken at temperature $T=4.8 \mathrm{~K}$. (b) The $J_{\mathrm{C}}$ vs. $T$ dependence with the maximum $8 \mathrm{MA} / \mathrm{cm}^{2}$ value marked. The inset shows $R-T$ characteristics for the same sample.

Figure 2 presents a current-voltage $(I-V)$ characteristics and $J_{\mathrm{C}}(T)$ dependence measured for the above structure. In Fig. 2a we show the $I-V$ curves thus below $T_{\mathrm{C}}$ and at the lowest studied temperature (inset), which was limited by DC current bias unit. We note that all our characteristics exhibit a very sharp superconducting-to-resistive transition with no measurable flux-flow region. In Fig. 2b, we demonstrate that for our best, $18 \mathrm{~nm}$ thick NbN film, $J_{\mathrm{C}}$ reaches $8 \mathrm{MA} / \mathrm{cm}^{2}$ at $T=4.8 \mathrm{~K}$, which is one of the highest values reported in literature, as, typically, $I_{\mathrm{b}}$ tends to be reduced by lithography process of the stripe patterning and some substrate flatness imperfections $[10,11]$. We also note that even at $T=14.5 \mathrm{~K}$, $J_{\mathrm{C}} \approx 0.2 \mathrm{MA} / \mathrm{cm}^{2}$. The inset in Fig. $2 \mathrm{a}$ shows the resistive transition $(R-T)$ of the same film, indicating that $T_{\mathrm{C}} \approx 15 \mathrm{~K}$.

\subsection{TEM studies of $N b N$ films}

A micrograph of a TEM cross-section of our $18 \mathrm{~nm}$ thick NbN film deposited on sapphire is presented in Fig. 3. A platinum layer visible on the top serves as a protection, used during the TEM specimen preparation. The cross-section shows an epitaxial growth of our film with the fcc structure, as well as atomically flat and very sharp interface. Some differences in the contrast levels of atomic-plane lines may suggest existence of oval grains at the upper part of the film. These were also observed in our previous AFM surface studies [8], as convex bows and can explain as the surface roughness after the RTA annealing. However, annealing at $1000^{\circ} \mathrm{C}$ clearly improves the film crystallization process, and, in consequence, leads to the reported high values of both $J_{\mathrm{C}}$ and $T_{\mathrm{C}}$.

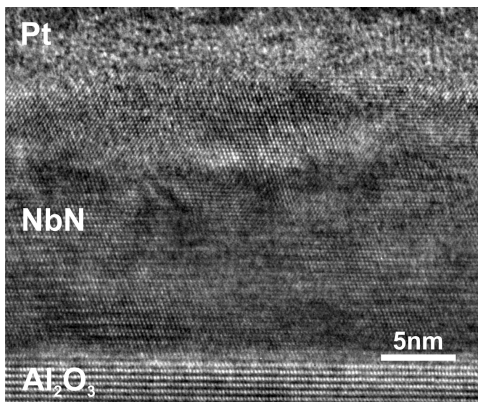

Fig. 3. A TEM cross-section of our $\mathrm{NbN}$ film indicating high level of epitaxy in the film.

\subsection{Superconducting and structural properties of $\mathrm{NbTiN}$ films}

We have studied transport properties of a nominally $20 \mathrm{~nm}$ thick NbTiN film deposited from separate $\mathrm{Nb}$ and $\mathrm{Ti}$ metallic targets (power $220 \mathrm{~W}$ and $80 \mathrm{~W}$, respectively) in the gas flow mixture $\mathrm{N}_{2} / \mathrm{Ar}$ of the total pressure of $8 \mu \mathrm{bar}$. Figure 4 shows the $R-T$ characteristics before and after the RTA process, indicating the increase of $T_{\mathrm{C}}$ from $9.5 \mathrm{~K}$ up to $13 \mathrm{~K}$ after annealing at $1000^{\circ} \mathrm{C}$ in Ar, the effect similar to that earlier observed in $\mathrm{NbN}$ films [8].

We have also performed extensive structural characterization studies of our NbTiN films and compared them directly to those earlier done for the NbN films [8]. X-ray 


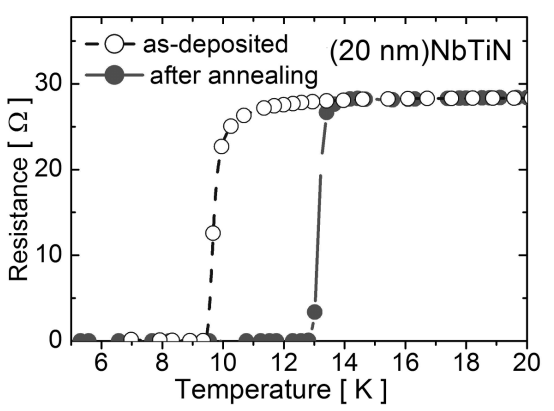

Fig. 4. The $R-T$ dependence for a $20 \mathrm{~nm}$ thick NbTiN film deposited on a sapphire substrate before and after the RTA process performed in $\mathrm{Ar}$ at $1000^{\circ} \mathrm{C}$ for $20 \mathrm{~min}$.

characterization was performed using the Pixcel detector working in a stripe mode. The resulting $2 \theta / \omega$ scan for the 111 reflection is shown in Fig. 5a. The scan has a highly symmetrical shape and reveals the characteristic "thickness" oscillations that indicate the actual film thickness of $15.3 \mathrm{~nm}$ and a good-quality film-substrate interface. The NbTiN lattice parameter $a=4.37 \AA$ was calculated from the 111 peak position, assuming a relaxed cubic lattice unit. The value is very close to the $4.368 \AA$ value previously obtained for as-deposited $\mathrm{NbN}$ [8], but it is lower than $4.389 \AA$ reported for NbTiN by others [11]. We believe that the latter might be due to a nonuniform substitution of smaller Ti atoms into Nb positions. The rocking curve (not shown here) of the 111 reflection measured with the analyzer resulted in the full width at half maximum $(\mathrm{FWHM})$ of $10 \operatorname{arcsec}(\approx 0.05 \mathrm{mrad})$, which was at the resolution limit of our apparatus and indicated an excellent crystalline quality of our specimen.
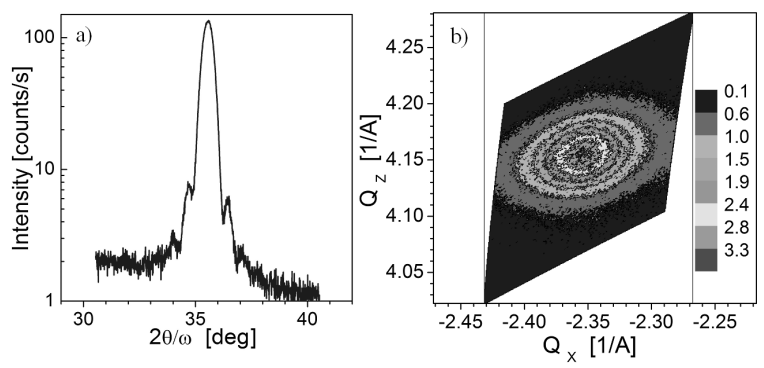

Fig. 5. The $2 \theta / \omega$ spectrum of the 111 reflection of a studied NbTiN film. (b) The RSM image of the 311 NbTiN Bragg reflection. The diagram represents intensity scale in the range of $0.1 \div 3.3$ counts $/ \mathrm{s}$. The maxima of intensities are located at center of the pattern.

The applied detector mode allowed to collect independently our NbTiN film, an asymmetric reciprocal space map (RSM) of the 311 Bragg reflection and establish, if the lattice unit cell of the crystal was deformed or not. The RSM image shown in Fig. 5b proves perfect ordering of the (311) plane and confirms that the NbTiN film grown by us is of high quality. Finally, the AFM studies (see Fig. 6) performed on the thinnest, $6 \mathrm{~nm}$ film

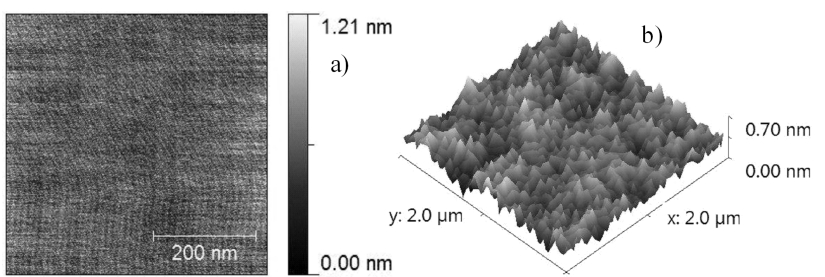

Fig. 6. AFM images of $(6 \mathrm{~nm}) \mathrm{NbTiN}$ film deposited on sapphire and RTA processed at $1000{ }^{\circ} \mathrm{C}$ in Ar for $20 \mathrm{~min}$.

indicated on a very smooth surface with the roughness of $0.1 \mathrm{~nm}$ even after the RTA processing. There are no sharp, spike-like structures as were observed in $\mathrm{NbN}$ films [8].

\section{Conclusions}

We have demonstrated that $18 \mathrm{~nm}$ thick $\mathrm{NbN}$ films grown on a sapphire substrate at optimized sputtering and annealing conditions are essentially epitaxial and exhibit excellent superconducting transport properties with $T_{\mathrm{C}}$ as high as $15.0 \mathrm{~K}$ and $J_{\mathrm{C}}$ reaching $8 \mathrm{MA} / \mathrm{cm}^{2}$ at $4.8 \mathrm{~K}$. Our early fabrication and characterization studies of ultrathin NbTiN films grown by the co-sputtering process from $\mathrm{Nb}$ and $\mathrm{Ti}$ targets in reactive plasma confirm that indeed NbTiN has a very high potential for SSPD applications. The NbTiN films grow in an epitaxial manner and exhibit exceptionally high surface smoothness, even after RTA processing. After annealing optimization their superconducting transport properties should be as good as those of NbN.

\section{Acknowledgments}

The authors thank I. Zaytseva for her contribution in measurements. The work was supported in part by the European Regional Development Fund (Innovative Economy, POIG.01.01.02-00-108/09). R.S. thanks the Spanish Ministry of Education and the University of Salamanca for their financial support and hospitality during his one-semester-long research visit in Salamanca, Spain.

\section{References}

[1] G.N. Gol'tsman, O. Okunev, G. Chulkova, A. Lipatov, A. Semenov, K. Smirnov, B. Voronov, A. Dzardanov, C. Williams, R. Sobolewski, Appl. Phys. Lett. 79, 705 (2001).

[2] R. Sobolewski, A. Verevkin, G.N. Gol'tsman, A. Lipatov, K. Wilsher, IEEE Trans. Appl. Supercond. 13, 1151 (2003).

[3] A. Verevkin, J. Zhang, R. Sobolewski, A. Lipatov, O. Okunev, G. Chulkova, A. Korneev, K. Smirnov, G.N. Gol'tsman, A. Semenov, Appl. Phys. Lett. 80, 4687 (2002). 
[4] A. Korneev, P. Kouminov, V. Izbenko, G. Chulkova, K. Smirnov, B. Voronov, G.N. Gol'tsman, M. Currie, W. Lo, K. Wilsher, J. Zhang, W. Slysz, A. Pearlman, A. Verevkin, R. Sobolewski, Appl. Phys. Lett. $\mathbf{8 4}$ 5338 (2004).

[5] W. Słysz, M. Węgrzecki, J. Bar, P. Grabiec, M. Górska, V. Zwiller, C. Latta, P. Bohi, I. Milostnaya, A. Korneev, O. Minayeva, G. Chulkova, K. Smirnov, B. Voronov, G. Gol'tsman, A. Pearlman, A. Cross, I. Komissarov, A. Verevkin, R. Sobolewski, Appl. Phys. Lett. 88, 261113 (2006).

[6] J. Chen, J.B. Altepeter, M. Medic, K.F. Lee, B. Gokden, R.H. Hadfield, S.W. Nam, P. Kumar, Phys. Rev. Lett. 100, 133603 (2008).

[7] M.T. Jarvi, M.J. Niedre, M.S. Patterson, B.C. Wilson, Photochem. Photobiol. 82, 1198 (2006).

[8] W. Słysz, M. Guziewicz, M. Borysiewicz, J.Z. Domagała, I. Pasternak, K. Hejduk, W. Rzodkiewicz, J. Ratajczak, J. Bar, M. Wegrzecki, P. Grabiec, R. Grodecki, I. Wegrzecka, R. Sobolewski, Acta Phys. Pol. A 120, 200 (2011).
[9] M.G. Tanner, C.M. Natarajan, V.K. Pottapenjara, J.A. O'Connor, R.J. Warburton, R.H. Hadfield, B. Baek, S. Nam, S.N. Dorenbos, E. Bermúdez Ureña, T. Zijlstra, T.M. Klapwijk, V. Zwiller, Appl. Phys. Lett. 96, 221109 (2010).

[10] N. Marrocco, G.P. Pepe, A. Capretti, L. Parlato, V. Pagliarulo, G. Peluso, A. Barone, R. Cristiano, M. Ejrnaes, A. Casaburi, N. Kashiwazaki, T. Taino, H. Myoren, R. Sobolewski, Appl. Phys. Lett. 97, 092504 (2010).

[11] J.R. Gao, M. Hajenius, F.D. Tichelaar, T.M. Klapwijk, B. Voronov, E. Grishin, G.N. Gol'tsman, C.A. Zorman, M. Mehregany, Appl. Phys. Lett. 91, 062504 (2007). 